\title{
Globe
}

Revue internationale d'études québécoises

\section{La voix du fils}

Le catholicisme au service d'une historicité de la langue dans Le Saint-Élias de Jacques Ferron

\section{The Voice of the Son: Catholicism in Service of a Historicity of Language in Jacques Ferron's Saint-Élias}

\section{Stéphane Inkel}

Volume 10, numéro 1, 2007

URI : https://id.erudit.org/iderudit/1000084ar

DOI : https://doi.org/10.7202/1000084ar

Aller au sommaire du numéro

Éditeur(s)

Globe, Revue internationale d'études québécoises

ISSN

1481-5869 (imprimé)

1923-8231 (numérique)

Découvrir la revue

Citer cet article

Inkel, S. (2007). La voix du fils : le catholicisme au service d'une historicité de la langue dans Le Saint-Élias de Jacques Ferron. Globe, 10(1), 147-170.

https://doi.org/10.7202/1000084ar
Résumé de l'article

Jacques Ferron, on l'a souvent remarqué, recourt à plusieurs reprises à des figures issues d'un lexique proprement catholique que ses contemporains tentaient pour leur part de mettre à distance. Au-delà de la " théorie du moi " et du rapport aux autres qui justifient, dans $\mathrm{Du}$ fond de mon arrière-cuisine, l'emploi « syntaxique » de cette terminologie, se peut-il que cet usage soit aussi le signe d'une épistémologie qui aurait survécu au processus de laïcisation de la Révolution tranquille? Cette lecture du roman Le Saint-Elias montre comment il est possible de déceler une structure de l'utopie messianique, privée de son contenu manifeste d'une " vocation » catholique en terre d'Amérique, aussi bien dans l'écriture de Ferron qu'au sein de ce que l'on appellera l'historicité québécoise. Une temporalité messianique épousant celle de la langue mise en scène par un rapport à l'origine que l'inversion de l'énoncé sur la filiation - « c'est le Fils qui a engendré le Père » - nous permettra d'appréhender. 


\section{ÉTUDES LIBRES}

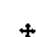

\section{LA VOIX DU FILS.}

\section{LE CATHOLICISME AU SERVICE}

D'UNE HISTORICITÉ

DE LA LANGUE

DANS LE SAINT-ÉLIAS

DE JACQUES FERRON ${ }^{1}$

\section{STÉPHANE INKEL}

Queen's University (Canada)

$+4++4+4+4+4+4+4++4+4+4+$

Résumé - Jacques Ferron, on l'a souvent remarqué, recourt à plusieurs reprises à des figures issues d'un lexique proprement catholique que ses contemporains tentaient pour leur part de mettre à distance. Au-delà de la " théorie du moi " et du rapport aux autres qui justifient, dans $D u$ fond de mon arrière-cuisine, l'emploi "syntaxique" de cette terminologie, se peut-il que cet usage soit aussi le signe d'une épistémologie qui aurait survécu au processus de laïcisation de la Révolution tranquille? Cette lecture du roman Le Saint-Elias montre comment il est possible de déceler une structure de l'utopie messianique, privée de son contenu manifeste d'une "vocation"

$$
+4
$$

1. Ce texte fait partie d'un projet de recherche intitule « Historicité er messianisme. Polinque et figures de la folie dans les manuscrits "Le pas de Gamelin" et "La grande tribu" de Jacques Ferron er Victor-Levy Beaulieu x, subventionné par le fonds FQRSC. 
catholique en terre d'Amérique, aussi bien dans l'écriture de Ferron qu'au sein de ce que l'on appellera l'historicité québécoise. Une temporalité messianique épousant celle de la langue mise en scène par un rapport à l'origine que l'inversion de l'énoncé sur la filiation - "c'est le Fils qui a engendré le Père" - nous permettra d'appréhender.

$$
\text { * * * }
$$

\section{The Voice of the Son: Catholicism in Service of a Historicity of Language in Jacques Ferron's Saint-Elias}

Abstract - It is often noted that on several occasions Jacques Ferron made use of personae originating in a proper Catholic vocabulary, a vocabulary from which his contemporaries tried to distance themselves. Is it possible that his use of these devices, beyond the "theory of the self" and the relationship to others who justify the "syntaxic" use of this terminology in Du fond de mon arrière-cuisine, was indicative of an epistemology that would have survived Quiet Revolution's secularization process? This reading of the novel Saint-Elias would like to show how it is possible to detect a structure of messianic utopia in Ferron's writing apart from its overt content of a Catholic "vocation" on American soil, as well as within what one would term Québécois historicity. Directed by a relationship to the origin that the inversion of filiation - "It is the Son who begat the Father" - allows us to understand, a messianic temporality adopts the temporality of language. $++4+4++4+4+4+4+4+4+4+4$

Il faut être un peu fou pour écrire. Comment expliquer autrement qu'un homme veuille se faire un nom avec les mots de tout le monde?

Jacques FERRON,

Du fond de mon arrière-cuisine

Jugeant que la nécessaire conscience de soi d'une société repose sur le "dédoublement de son être empirique et de son être symbolique ${ }^{3}$ ", le sociologue et philosophe Fernand Dumont a consacré l'un de ses derniers ouvrages à l'analyse du processus d'une conscience de soi qui, au Québec, aurait subi d'importantes mutations suite à la Révolution tranquille. Si la conscience religieuse est loin d'être la seule condition d'un être symbolique, il n'en demeure pas moins qu'elle fut longtemps, avec la langue, la composante essentielle de la référence de cette conscience de soi, comme l'a montré Dumont dans Genèse de la société québécoise. On se souviendra ainsi

$$
+4
$$

2. Jacques Ferron, Du fond de mon arrière-cutsine, Montréal, Éditions du jour, 1973, p. 46.

3. Fernand DUMONT, "Jadis, une société religieusen, Le sort de la culnure, Montréal, Typo, 1995, p. 299. 
que cet ouvrage entendait penser les conditions d'une nouvelle conscience de soi en effectuant la genèse de la précédente $e^{4}$ dont le décès avait, dans une conférence de 1985, conduit le sociologue à cette question inquiète:

S'il est vrai que cette société fut religieuse, ce n'est pas seulement le rôle de l'Église qui s'est effondré au cours des décennies récentes; du même mouvement, une société a perdu sa traditionnelle conscience de soi, et sans qu'elle en ait trouvé une autre [...] comment, [dès lors], envisager l'avenir'?

Si la référence religieuse n'a toujours pas été remplacée, la langue apparaissant désormais comme le seul dénominateur commun d'une identité de plus en plus indéterminée, et ambiguë - comme l'appréhension fréquente de la culture québécoise par le filtre de la francophonie le montre aisément -, il me semble que la question elle-même de Fernand Dumont mérite d'être reformulée. Car si "l'effondrement" de la société religieuse d'hier se décline sur au moins trois points - perte d'influence de l'Église; foi de plus en plus diffuse et intérieure, si ce n'est absente; migration de la vie sociale de la paroisse vers différents pôles de la société civile (syndicats, organisations non gouvernementales, chambres de commerce, etc.) - un autre aspect de la conscience religieuse, insuffisamment pris en compte, demeure encore repérable au sein de notre société. Il s'agit de ce que l'on pourrait appeler une épistémologie catholique qui continue d'habiter la conscience de soi de la société québécoise, lisible aussi bien dans le partage d'une expérience de la temporalité que dans le rapport à la langue. C'est ce rapport à la langue, anthropologiquement catholique, donc, que l'on retrouve énoncé, voire théorisé, dans un chapitre bien connu de l'essai $D u$ fond de mon arrièrecuisine de Jacques Ferron. Car s'il y a un écrivain québécois qui a su lier la question linguistique avec celle de l'héritage, ici plus qu'ailleurs religieux, c'est bien Ferron, lui qui dans ce livre fait du dogme de la Trinité une affaire de syntaxe, et qui au surplus n'a cessé de ramener la figure du Christ - qu'il identifie toujours à une posture de Fils - à l'intérieur de ses principaux romans. Que faut-il penser de la récurrence d'une telle figure? Se peut-il qu'elle témoigne d'un rapport à la langue qui ait tout à voir avec la référence québécoise - entendue ici selon la définition de Fernand Dumont d'une

$$
++4
$$

4. Fernand Dumont, Genise de la soritzt québtcoise, Montréal, Boréal, 1993, p. 13-14: "Lorsque s'effrice l'idencité collective, ne faut-il pas se demander par quel processus elle s'écait imposte autrefois, revenir à sa genese, si l'on veut parvenir à une nouvelle conscience de soi? "

5. Fernand DUMONT, «Jadis, une sociéré religieusen, p. 289. 
représentation, ou conscience de soi, partagée collectivement -, tout en offrant une posture éminemment singulière permettant au sujet de s'inscrire en tant que sujet à l'intérieur de la communauté? Questions complexes que j'aimerais à tout le moins circonscrire en relisant le roman déterminant quant à cette question de la filiation, Le Saint-Élias, qui nous met directement aux prises avec l'intrication de ce problème de la parole et de la figure du Fils.

Avant d'aborder ce texte et les développements qui lui font signe au cour de l'essai $D u$ fond de mon arrière-cuisine, sans doute faut-il au préalable s'arrêter sur un court passage de l'un des "contes d'adieu" de $L a$ conférence inachevée qui nous donne pour ainsi dire l'ultime position de l'écrivain sur son rapport à la langue. À l'intérieur du conte "Adacanabran", texte autobiographique à peine voilé où le docteur Legris, immobilisé par une tempête dans un village d'Abitibi, raconte à ses jeunes compagnons d'infortune son voisinage avec la folie qui l'a ainsi relégué, à un âge avancé, à un dispensaire de province, Ferron reprend la dialectique du "moi" et de la langue sous la figure d'une "fêlure à la tempe gauche $e^{6}$. Mais une étrange fêlure, en vérité, puisqu'elle permet aussi bien à son esprit de se répandre dans le monde afin de "l'inspirer?" qu'elle apporte au docteur Legris un "irrépressible besoin de pervertir le langage, ce bien commun indispensable, d'en altérer la forme pour en couler le fond ${ }^{8}$ ". On peut s'étonner de la poétique d'un tel énoncé, qui semble à mille lieues de la prose cristalline d'un écrivain qui s'est à maintes reprises prononcé sur la nécessité de la politique afin de protéger une langue qui était menacée 9 . À même ce paradoxe, il est possible de déterminer un rapport à la langue qui ne va pas de soi et qui, loin d'être aussi transparent que Ferron le laisse parfois entendre, est au contraire affaire de division et de rupture, d'ellipses et de rapport à l'Autre. Ce rapport ne se résout jamais, comme les nombreux fantômes de l'œuvre nous le prouvent aisément. Entre le sujet et les autres, il y a une distance infranchissable, un abîme, que même une langue en partage ne parvient pas à lier. Plutôt que d'y voir un processus de déréliction qui aurait d'ailleurs fini par avoir raison de l'écrivain, peut-être faut-il déceler dans cette figure de la fêlure une véritable politique d'énonciation s'opposant en tous points à la nécessaire défense, politique elle aussi, mais d'un autre point de vue: celui d'une langue menacée. Au-delà de l'aspect historique et identitaire de la langue, telle qu'on la retrouve chez la

$$
+++
$$

6. Jacques Ferron, La conférence inachevée, édirion préparée par Pierre Cantin, Marne Ferron et Paul LewIS, Montréal, VLB, 1987, p. 208.

7. Ibid., p. 213: «Par la fàlưre, mon esprit s'étaic dispersé, inspirant le monde entier. Quel prodige! »

8. Ibid, p. 209.

9. Voir Jacques Ferron et Pierre L'Herault, Par la porte d'en amère. Entretiens, Montréal, Lanctôt, 1997, p. 47. 
plupart des contemporains de Ferron, ce serait sa dimension collective qui s'avérerait problématique pour l'écrivain. D'où la nécessité de s'y inscrire au scalpel, de la diviser de l'intérieur, à coup de "fêlure" ou de crucifixion, et de constamment remettre sur le métier non pas tant l'œuvre à faire que la position que le sujet y occupe, à la fois dédoublée et séparée ${ }^{10}$, à l'image de ce Dr Legris/Adacanabran qui à l'aide de sa petite fêlure imaginaire à la tempe gauche joue le rôle d'agent double au sein de la langue, en usant à distance, au point d'en être la victime aussi bien que le contempteur.

\section{UNE SYNTAXE TRINITAIRE}

Si l'on exclut "Les salicaires" - qui, avec l'«Appendice aux Confitures de coings" paru un an auparavant, inaugure la veine autobiographique qui dominera l'ensemble de la dernière période d'écriture de Ferron -, le texte le plus intéressant du recueil d'essais $D u$ fond de mon arrière-cuisine est sans contredit "La descente de la croix selon Monsieur Camus, auteur de L'Étranger", où il est au demeurant assez peu question de "Monsieur Camus». On se souviendra que ce texte est en partie constitué de "fragments de thèse" que Ferron affirme avoir soutenue à l'Université McGill «sur le moi décrucifié, tombé de la croix dans une soupière pleine, catholique et québécoise" ", et dont il reconstitue dérisoirement la scène, "théorie du moi" empruntant explicitement au vocabulaire religieux, alors en phase accélérée d'effacement du discours public. Ce n'est évidemment ni par simple souci de préserver la mémoire d'un discours jusque-là prépondérant ni par plaisir de la contradiction que Ferron emprunte ainsi à ce vocabulaire. C'est surtout que le discours catholique, avec ses dogmes et ses personae à la surface signifiante toujours vive, permet encore - mais pour combien de temps, voilà déjà l'une des questions implicites d'un tel texte - à l'écrivain d'inscrire au sein de sa scénographie prétablie de tout autres questions, que l'on dira relatives à la filiation. Qu'est-ce que parler en tant que fils? Quelle place occupent les autres? Et surtout, d'où me vient ce Verbe dont j'ai la garde, mais qui me lie également à ces autres dont je ne sais rien? Après avoir décrit, dans l'"Appendice aux Confitures de coings", comment son père "avait une difficulté d'élocution" qui lui faisait casser le français "comme s'il eût parlé

$$
++
$$

10. Voir, à cet égard, la figure dédoublée du narrateur dans le manuscrit Le pas de Gamelin, séparée entre Nocaire ec Maski, et la tentative de résolution qui en découle dans L'exécution de Maski (jacques Ferron, Rosaire précédé de L'execution de Maski, Moncreal, VLB, 1981). Des extraits du Pas de Gamelin, à ne pas confondre avec le texte "Le pas de Gamelin " publié dans La conference inachevee, ont été publies et presentés par les soins de Pacrick POIRJER, dans Ginecte Michaud [éd.], L'autre Ferron, Montréal, Fides - CETUQ, "Nouvelles études québécoises " 1995. 11. Jacques FERRON, Du fond de mon amiere-cusine, p. 170. 
surtout l'anglais qu'il ne parlait pas ou guère", Ferron écrit: "j'ai pris le parti d'écrire, seul et me retirant du monde, bien obligé à la longue de réfléchir. Je le fais souvent à propos de ma mère, tôt partie et qui m'a par conséquent intrigué, mais je reste fidèle au patronyme ${ }^{12}$." On voit bien comment l'écriture, chez Ferron, est située dans un espace d'entre-deux que ses derniers romans, inachevés, cherchent à délimiter, entre l'héritage du "patronyme», legs d'une figure paternelle qui pour l'écrivain aura représenté "La Plus Haute Autorité ${ }^{13}$ ", et une figure de la mère. Cette dernière, partie trop tôt, n'a pas de limites identifiables, mais se confond bien souvent avec la langue elle-même et la culture française que son éducation chez les Ursulines lui aura transmise, avant de la léguer à son tour à son fils, notamment par le biais du prénom $^{14}$. Mais si Ferron se dit "fidèle au patronyme", c'est bien la figure de la mère qui sans cesse revient le hanter. Lorsque Ferron emprunte au dogme de la Trinité pour définir sa posture au sein de la langue, c'est donc à une opération de transposition qu'il s'adonne; une transposition ou une traduction, dans un régime symbolique lié à la loi du Père, qu'il s'agit pour nous de comprendre comme un processus de mise à distance du sujet envers le fantôme maternel, si ce n'est de l'élément proprement commun de la langue ${ }^{15}$. Commentant l'utopie de Port-Royal visant à réduire la "confusion"

$$
+4
$$

12. Jacques FERRON, "Appendice aux Confitures de coings ou Le congédiement de Frank Archibald Campbell", Les confirures de coings et autres textes, Montreal, Part pris, 1972, p. 275-276. Voir Egalement l'historiette "Notaire par le nez», publiée dans Jacques FERRON, Papiers intimes. Fragments d'un roman familial: lettres, bistorzettes et autres textes, édition préparée et commentée par Ginette MiCHaud et Pacrick POIRIER, Outremont, Lanctôt, "Cahiers JacquesFerron", nos 1-2, 1997, p. 313 : "Ex puis, un jour, ma mère mourut et me devint plus précieuse."

13. Voir, abtd., l'avant-propos de Ginette Michaud, qui explıque comment les historiettes de ces Papiers tntumes, certaines pubhées dans L'Information médicale et paramédicale, en 1975 et 1976, étaient au départ destinées à deux projets abandonnés par Ferron, l'un sur la figure du père et devanc s'intituler la Plus Haute Autorsté er l'aucre sur la figure de la mère, et la famille Caron en général: la Miss et sa sceutr, ma mère. Madeleine Ferron, sceur de l'écrivain, a d'ailleurs indiqué comment c'est la recherche de Jacques sur la famille de la mère qui est à l'origine de sa propre évocation dans Adrzenne. Une saga familiale (Montréal, Boréal, 1993.). Ce n'est donc pas un, mas trois romans (avec Le pas de Gamelin) que Ferron aura envisagés autour de 1975 et qu'il aura abandonnés à des degrés d'avancement divers.

14. Sur la notion de langue maternelle, certe langue du roman où le fanţôme de la mère ne cesse de surgır, je me permets de renvoyer à ma thèse de doctorat: " Les fancômes et la vox. Politique de l'aliénation et langue maternelle chez Rejean Ducharme et Samuel Beckett ", Université du Québec à Montréal, 2005. Quant à la question du prénom, voir l' "Appendice aux Confirures de coingsn, qui raconte comment la mère de l'écrivain, la veille de sa mort, lur "demanda de changer [son] prénom de Jean-Jacques en celui de Jacques er de "ne pas [se] penser plus fin que les autres" " (p. 282). Le prénom Jean-Jacques faisant ici référence à Rousseau, on peut se demander ce qu'ımplique comme rapport à la culcure françaıse de s'en voir subicement privé. Sur les implications de ce changement de prénom comme héritage de la mère, voir Patnck POIRIER, « Feu Jean-Jacques, ou le legs maternel ", Papiers intimes, op cit, p. 119-166.

15. "Le meilleur moyen pour évirer la confusion des mots qui se rencontrent dans les langues ordinaires est de faire une nouvelle langue et de nouveaux mots qui ne sotent atrachés qu'aux idtes que nous voulons qu' ils représentent; mais, pour cela, il n'est pas nécessaire de faire de nouveaux sons, parce qu'on peur se servir de ceux qui sont déjà en usage, en les regardant comme s'ils n'avaient aucune signification, pour leur donner celle que nous voulons qu'ils aient ", La logique de Port-Royah cité par Jacques FERRON, Du fond de mon amere-cusstne, p. 45. Voir également rel extrait du manuscrit non publié Le Pas de Gamelin, cuté par Ginetre Michaud, où l'on retrouve Maskı au camp militaire de Grande-Ligne ergotant sur la solitude "en langue* qui lui permet la garde de prisonniers allemands au milieu d'officiers anglophones: «Ici, au 
des mots de la "langue ordinaire», Ferron peut alors écrire, rendant justice à l'équivoque, que les «mots, appris durant la première enfance, sont bien plus que des signes rationnels et qu'avant de parler en termes on parle pour parler, par plaisir ${ }^{16}$ ". Prendre place dans un régime de signes et reconnaître sa dette envers les figures qui ont pu présider à cette entrée, voilà, somme toute, ce qui amène Ferron à investir la mémoire d'un discours théologique afin de s'en servir comme paravent, voire théâtre, dans sa tentative de remontée des origines.

La première chose que l'on peut noter, c'est que la figure de "Dieu", dans le texte de Ferron, intervient en tant qu'intercesseur, "truchement", entre le "moi» et les autres. Il obtient ce titre par sa faculté, intrinsèque à la théologie trinitaire, de se "dédoubler", régnant à la fois sur ce que Ferron appelle le «Paradis" (soit l'éternité propre à la "prolifération de l'espèce ${ }^{17}$ ), tout en donnant "un sens à la vie" en ayant vécu "son agonie dans le jardin des Oliviers": "participant de l'un et de l'autre, il est la langue commune par laquelle l'inconciliable se rencontre et l'unique au monde, que la mort ronge du dedans, communique avec tous les autres que soi, que la vie multiplie par le dehors ${ }^{18} . "$ Au-delà de tout rapport de croyance ${ }^{19}$, Ferron a donc recours au dogme trinitaire afin de penser une position dans la langue qui serait à la fois unique, à l'image du Fils «abandonné de tous dès le jardin des Oliviers, principe de la mort individuelle, toujours solitaire ${ }^{20}$ ", et partagée, puisqu'elle fait appel à la chose même qui crée du même, une communauté. Mais il s'agit aussi d'une solution de continuité, si l'on en croit le recours à la syntaxe qu'effectue simultanément Ferron, selon une logique scolastique somme toute des plus classiques. Lorsqu'il fait intervenir ces considérations de syntaxe, ce n'est plus du tout de la spéculation, mais le nœud d'un problème faisant du rapport aux autres - qui est surtout, dans le cas de Ferron, rapport à l'Autre ${ }^{21}$ : à Dieu, à la loi du patronyme, au fantôme

$$
++4
$$

milieu de mes Old Vets, je n'ai jamaıs été aussi seul avec moi-mème ec content de l'être, loin du Nous coercitif, cette première personne du pluriel er de la cohésion, cette voix de mon pays", Ginette MICHAUD, *De Varsovie à GrandeLigne: l'ceuvre in extremis", Littératures, $n^{\infty} 9-10,1992$, «Présence de Jacques Ferron ", p. 98. Voir également la nore 47 où elle souligne un lapsus de Ferron, qui écrit "cohersirif" à la place de "coercitif», soulignanr du même coup le carcan que représente pour lui la cohésion d'une langue marernelle.

16. Jacques FERRON, Du fond de mon amiere-cuistne, p. 45-46.

17. Ibid, p. 151.

18. Ibid, p. 151-152.

19. "Ils sont très nombreux et je suis seul; ils proliferent et je me meurs. Tout cela implique des conséquences; par exemple je commence à avoir idée de Dıeu comme truchement nécessaire. Une idée qui m’ennuya beaucoup, vu que je me crois athée er que je n'ai jamais ménagé le curés (ibid, p. 144).

20. Jacques FERRoN, "Appendice aux Confirures de coings", p. 315.

21. Il y aurait beaucoup à dire sur certe question des "autresn, dans le cexce de Ferron. Car en insistant sur l'impossibilité d'entrer en relation avec eux autrement qu'en supposant «que les autres avaient, eux aussi, un perit studio d'artiste dans 
de sa "mère cadette ${ }^{22}$ " - le point de rencontre des questions sur la langue et sur le temps, sur la syntaxe, précisément, et l'organisation temporelle dont elle est le soubassement:

dans une conversation où les mots passent l'un après l'autre dans une suite linéaire où la place de chacun lui confere un rôle, qui le nuance et le précise, dans la phrase, première instance du discours, une place de sujet, de verbe ou de complément, ce qui le réduit, avant toute compréhension, à n'être qu'un instant, presque intemporel, telle la seconde que marque en sautillant la trotteuse d'une montre, entre le passé des heures, des jours, des années et des siècles, et l'avenir aussi long par devant, [...] Dieu laisse au présent son évidence, sa phonème [sic], son mot et compose la phrase, le paragraphe, le livre sur une durée plus longue, incluant le passé et le futur [...] on le qualifie d'Éternel à cause de cette pérennité, des trois temps qu'il domine et de l'oiseau qu'il entretient dans le ciel, au-dessus d'un vaste espace existentiel, qui, sous le nom d'Esprit-Saint, dans le genre fou de Bassan, vole sans cesse et qui, de temps à autre, plonge dans la mer et l'oubli pour aller retrouver le mot qui manque dans la suite du discours et risquerait d'interrompre monologues et entretiens dans lesquels le verbe se complait $[s i c]$ et continue son procès d'engendrement commencé avec saint Jean pour le plus grand profit de l'homme ${ }^{23}$.

C'est bien "l'homme" qui est ici en question dans ce recours à Dieu, l'homme, c'est-à-dire le sujet, au sens extrêmement précis du terme que lui ont donné aussi bien Lacan que Benveniste dans leur théorie respective de

$$
+4
$$

les cheveux", "pure hypothèse et alıénation réciproque" (Du fond de mon arrière-cutstne, p. 141), ajoute Ferron, l'ecrivan désigne en fait le silence de l'Aurre nécessaire à la constiturion de sa propre subjectivité, pure transcendance que l'on croirait directement tiré des Écrits de Lacan. Mars la question n'est pas de mesurer le degré de confluence de la théorie ferronienne avec relle ou telle épıstémologıe, ni mème de déterminer l'étendue de sa cohérence, mais bien de voir comment c'est la question structurante de la langue du sujer qui intervient au sein de ce développement spéculatif. Il ne s'agit donc pas de paraphraser le texce de Ferron, ni même de le récuser - Anne CAUMARTIN a récemment souligné le malaise habituel de la critique envers ce texte (" Réecriture er muance chez Jacques Ferron *, Brigitte FAVVRE-DUBOZ et Parrick POIRIER [éd.], Jacques Ferron: le palimpseste infinz, Outremont, Lanccôt édiceur, "Cahiers Jacques-Ferron ", $\mathrm{n}^{\text {os }}$ 89, 2002, p. 376) - mais plus simplement d'en déceler les enjeux poécıques, voire politiques.

22. Expression que l'on va souvent retrouver à partir de l'kAppendice aux Confitures de coingsı (p. 325), notamment dans certaines des historiettes destinées au double roman familial de l'auteur, de même que dans La conférence inachevee, er que Ferron emploie pour souligner le jeune âge de sa mère au moment du décès. On encend bien dans une celle expression comment la mère est d'abord une figure de temps, éloignée par la distance temporelle, mais aussi lieu d'une chronologie renversée où c'est le fils qui devient l'âné (à défaut de l'«engendrer», selon la figure du Satnt-Élias que l'on va bientôt analyser) de sa propre mère. Sur la relation, parfois tourmentée, qu'entretient Ferron avec le souvenir de sa mère, on consultera l'essai biographique de Marcel OLSCAMP, Le fils du notaire. Jacques Ferron (1921-1949) (Fides, 1997, notamment p. 41,71-72), où l'auteur rend compre de l'héritage à la fois linguistıque et littéraire de la mère, cerce «langue trop belle " qui deviendra paradoxalement la * langue littéralement maternelle de l'écrivain *.

23. Jacques FERRON, Du fond de mon amerencuisine, p. 152. 
l'énonciation. Quelle opération "Dieu» vient-il ici effectuer? Il semble qu'il vienne avant tout remplir l'ellipse pleine d'angoisse du sautillement de la trotteuse. "Rejointant" le "moi" et les autres, pour utiliser un mot d' Hamlet sur lequel Ferron n'a cessé de réfléchir, il réunit également les éléments de la phrase dans le temps. En attribuant à "Dieu» le rôle du verbe, Ferron ne fait pas qu'insister sur la ligature propre à la langue, ontologiquement créatrice de collectivité. Il fait de lui, au sens linguistique du terme, le véritable sujet du discours, vecteur d'énonciation permettant à la "conversation" de suivre son cours et au mot de succéder au mot. Il autorise aussi le sujet de l'énoncé, ce "moi" prisonnier d'un studio, à céder le pas à un verbe qui le dépasse, qui n'est autre que ce sujet d'énonciation réconciliant le "moi" au lieu culturel du discours, ou, si l'on veut, la langue que l'écrivain a en partage. C'est ce que j'appellerai la voix: lieu d'intersection de la langue commune et de l'irréductibilité du sujet, elle est cette distance par rapport à l'espace immédiat du sujet produite par le recours à une mémoire qui ne lui est pas pour autant extérieure, mais simplement située dans son point aveugle, au sein même des mots chargés de sens qui, au dire même de Ferron, lui "imposent leur régime $e^{24}$.

Avant de voir comment un tel sujet et la voix singulière qu'il met en œuvre opèrent au sein du roman de Ferron et révèlent ce double rapport au temps et à la langue inclus dans le discours religieux le plus traditionnel dans une modernité encore inédite, il nous reste à poser un certain nombre de questions sur ce recours au religieux et sur l'arrière-fond politique qui y préside. Car c'est bien de cet usage qu'il est ici question. Comment "moi", spécimen unique, puis-je entrer en relation avec les autres en empruntant les mots d'une même langue? Avant d'accepter les énoncés de Ferron sur la figure du Verbe palliant tous les trous du discours, il faut peut-être se souvenir que la religion est une série de découpages et de séparations. Séparation du monde de l'instant et de l'éternité qui lui donne sens, mais aussi, d'une façon plus originaire, séparation d'un phénomène ou d'un objet de la sphère profane vers une sphère sacrée; soustraction, au sens propre, de tout objet, serait-il de langage, chargé d'une fonction de représentation pour un dieu, quel qu'il soit. Faire appel au langage de la théologie, en ce sens, c'est faire intervenir cet acte de découpage afin de retirer une parole du circuit qui lui donne sens et, par conséquent, d'ajouter à la valeur d'usage propre aux mots une tout autre valeur, symbolique, que l'on peut désigner avec Walter

$$
+4+
$$

24. 16 id, p. 146 . 
Benjamin comme la "communicabilite $e^{25}$ " inhérente au fait de parler. Le Saint-Élias s'applique à mettre en scène ce double usage de la parole, que l'on retrouve dans les modalités les plus diverses (rhétorique, théologique, politique), et donne encore à voir les tensions les plus vives qui animent la dialectique de cette voix en train de se constituer.

Parmi les forces qui animent cette tension de la voix, il faut évidemment compter sur la question identitaire qui traverse le roman en son entier; non seulement par l'entremise de sa figure éponyme, ce trois-mâts nommé le "Saint-Élias" en l'honneur du chanoine Élias Tourigny, construit afin de "briser l'écrou du golfe» et de faire des gens de Batiscan des "gens de toutes les mers du monde ${ }^{26}$, mais encore par le recours à la logique du métissage et de la filiation symbolique. Une identité dans laquelle interviendrait, encore une fois, l'action de la soustraction: "Québécois, c'est être ceci, cela, n'importe quoi avec quelque chose en moins. La soustraction est de rigueur ${ }^{27}$." Question de mots? Certes, mais avec quelque chose de plus... Il me semble que, par la seule mention de cet opérateur logique, Ferron parvient à saisir la mouvance dialectique d'un rapport au temps et à l'histoire qu'il convient de nommer politique ${ }^{28}$. Qu'est-ce donc, en effet, que ce "quelque chose en moins", si ce n'est la révocation de la fiction téléologique régnant sur la conception historiciste de l'histoire s'appuyant sur le mythe du progrès? Brossant l'histoire à rebrousse-poil, selon la célèbre image des thèses "Sur le concept d'histoire" de Walter Benjamin, Ferron s'est appliqué à relire l'une des époques les plus chargées de notre histoire, celle de la deuxième moitié du XIX siècle, afin d'ouvrir au sein de son discours dominant, celui-là même que ses contemporains s'empressaient d'oublier, des possibles qui seraient toujours à venir, voire qui n'auraient de valeur que par l'inachèvement qu'ils mettent en œuvre.

$$
+4
$$

25. Voir le célèbre énoncé qui résume la philosophie du langage du premier Benjamin, visant à souligner la porrée indicative du langage contre touce visé instrumentale: «Il n'y a pas de concenu de langage; comme communication, le langage communique une essence spirituelle, c'est-à-dıre purement et simplement une communicabilité*, Walter BENJAMIN, "Sur le langage en général et sur le langage humain ", CEuvres I, Paris, Gallimard, coll. « Folio", 2000, p. 150. 26. Jacques FERRON, Le Samt-Ellas, Montréal, Typo, [1972] 1993, p. 33.

27. Jacques FERRON, Du fond de mon amitre-cuistne, p. 180.

28. À propos de l'origine de la "théorie du moi», Ferron écrir qu'l a compris tardivement que "la politique écait secondaire et que prımait le rapport entre le moi et les autres" (Du fond de mon arrière-cuistne, p. 144). Si l'on serait porté à croire que Ferron confond ici $l a$ politique, telle qu'il a pu la pratiquer au sein du PSD, d'abord, et du Parti Rhinocéros, ensuite, et le poltrique, que l'on peur définir comme étant l'espace symbolique où se posent les rapports du sujet et de la communauté, ce serait faire $f$ du fair qu'il abandonne totalement la première pour se consacrer, à travers divers écrits, à cette question de l'inscription du sujec dans la collectiviré. 


\section{LA MËRE ET LE NOM}

$\mathrm{Si}$ je choisis Le Saint-Élias afin de lire et d'analyser ce rapport à la langue inscrit en toutes lettres dans le roman ferronien, c'est parce qu'il met en scène comme nul autre la parole en tant que parole. À cet égard, il n'y a qu'à ouvrir le livre pour la voir surgir, dûment mise en place par le décor qui en est l'occasion, le lancement du trois-mâts le "Saint-Élias", et même mise en "bouche ${ }^{29}$ ", pour ainsi dire, par Mgr Charles-Olivier Caron. C'est ce dernier, grand-oncle de l'écrivain, qui "parla le premier et, mine de rien, prépara le discours du curé de la paroisse, messire Élias Tourigny, qui n'était pas un orateur et qui, pour une fois, à la surprise de tous, comme par miracle, fut éloquent ${ }^{30}$ \%. Il y est donc question d'autorité, d'éloquence et de cette éloquence religieuse particulière au Québec du XIXe siècle, toujours liée à un contexte de réception, comme l'apprendra le pauvre abbé Lupien qui "malgré toute son éloquence [...] n'avait pas la même façon de parler que ses paroissiens ${ }^{31}$ \%. Cette première scène s'avère d'une grande complexité puisqu'elle met aux prises au moins deux conceptions de la parole. Celle de l'abbé Lupien, jeune vicaire sortant tout juste du séminaire et "qui avait de la passion pour la rhétorique ${ }^{32}$ ", qui assiste à la scène; et celle du chanoine Tourigny, "homme à chevaux" qui "s'il était bon curé [...] n'avait pas l'art de parler ${ }^{33}$ ". Voilà donc un "médiocre orateur", la chose est soulignée à trois occasions, qui malgré tout, "pour une fois", "à la surprise de tous", "comme par miracle", est à ce point éloquent que son vicaire, celui-là même qui l’a toujours jugé "fort médiocre" orateur, se demande si «l'Esprit saint [ne] serait [pas] descendu sur lui ${ }^{34}{ }$. Que faut-il comprendre de cette opposition? Ce registre du "miracle» et de l'exception n'est-il qu'une simple hyperbole servant à appuyer le discours du chanoine, pourtant des plus simple puisqu'il y est question du fleuve, d'un voilier et des gens de Batiscan? La chose serait déjà remarquable par son paradoxe, puisque Ferron ferait ainsi usage d'un lexique de l'exception propre au discours messianique traditionnel; celui de Mgr Laflèche, par exemple, que le discours de l'abbé Tourigny et de l'ensemble du roman cherche précisément à remettre en question par la

$$
+++
$$

29. Jacques FERRON, Le Saint-Elias, p. 29.

30. Ibid, p. 27-28.

31. Ibid, p. 64 .

32. Ibid, p. 30.

33. Ibid, p. 29.

34. Ibid, p. 30. Voir également p. 28 où un autre passage mappelle cette scène de la descente de l'Esprit saint, lors de la Pentecôte, où l'on se souviendra que les apôtres parlaient en langues, écant ainsi compris de chacun, étrangers inclus, comme s'ils parlaient "sa propre languen ("Actes des Apôtresn, 2, 6) : "ll dit alors des mots qui dejà venaient sur les lèvres de rous, ce qui éronna d'abord, puis parut prodigieux et suscita des cris enthousiastes." 
métaphore du trois-mâts, "bris[ant] l'écrou du golfe ${ }^{35}$ " afin d'ouvrir la population de Batiscan au reste du monde ${ }^{36}$. Au-delà de cet événement unique qu'est le discours du chanoine, deux conceptions de la langue s'opposent ici. Cette opposition qui ne se résout jamais constitue la tension au sein de laquelle le roman cherchera à poser les termes d'une tout autre équation.

La première conception est personnifiée par le chanoine, homme d'autorité dont l'ascendant sur ses paroissiens ne fait pas de doute, et qui tire précisément cette autorité du fait qu'il parle exactement comme eux ${ }^{37}$. Appelons cela l'être-en-compagnie de la langue. Nous pouvons, pour faire court, associer la seconde conception à la rhétorique, cette langue des livres pour laquelle se passionne l'abbé Lupien, mais qui l'empêchera paradoxalement de communiquer, si ce n'est communier, avec ses futurs paroissiens. Nous retrouvons dès lors les termes exacts qu'utilise Ferron dans les essais de Du fond de mon arrière-cuisine: la "soupière" d'une langue collective où chacun dit "des mots qui déjà venaient sur les lèvres de tous ${ }^{38}$ " et la Passion du "moi crucifiant" devant recourir au Verbe, c'est-à-dire à la langue des «autres", pour ne pas être seul, même s'il ne s'y retrouve guère, pliant plus volontiers la réalité à ses desseins, s'il en a les moyens, qu'enclin à s'y soumettre ${ }^{39}$.

$$
+4
$$

35. Jacques FERRON, Le Saint-Elias, p. 33.

36. Voir, sur l'opposition entre "verticalité" messianique et "horizontalıté" d'échange er de métissage, Pierre L'Hérault, à qui l'on doit le texte dérermınanc sur cette question identıaire dans "Le Sannt Élias: sauver l'enfanc", Lautre Ferron, op. cit., p. 100, 104: "Dans le contexte de la lutte du libéralısme ex de l'ultramontanisme, qui forme celui de l'histoire du Samt-Elias, la figure du mécissage repousse la vision génétique d'un peuple messianique, à tout jamais fixé dans un destin er une mission." La fin du discours du chanoine est de ce point de vue exemplaire: "Vous me demanderez pourquoi nous l'avons construit. Je vous répondrai que ç'a été pour briser l'ecrou de notre pays. Il écait bon de rester enfermés aussi longtemps que nous n'étions pas un peuple. Mais ce peuple, nous le sommes enfin devenus: que soit brisé l'écrou du golfe! que cessent les empêchements de l'enfance! [...] nous serons libres, nous serons gens de toutes les mers du monde. " (Le Saint-Élias, p. 33) Rappelons qu'il s'agit ici d'une thèse défendue de longue date par Ferron, de la pièce des Grands soletls jusqu'aux entretiens accordés à Pierre L'Hérault en 1982, voulant que la nationalité canadiennefrançaise ne débute qu'après les rébellions de 1837. Voir Par la porte d'en arrière, op. cit., p. 128-129. On peut malgré tout se demander ce que seraur le discours messianique privé des artributs de sa «mission». Il y aurait tout un cravail à accomplir, que nous ne pouvons ici qu'esquisser, afin de montrer comment Ferron ne commer pas une simple satire de l'ultramontanisme de Mgr Lafleche ec du discours messianique dont il est l'un des principaux fers de lance, mais qu'll cherche au concraire à le revivifier de l'intérieur, le nettoyane de ses scortes consolatrices - celles de l'utopie de la " mission" catholique en terre d'Amérique - mais conservant le rapport au temps et a l'histoire qui en est le fondement. Voir la dernière section ci-dessous.

37. On trouve d'alleurs un parallele histonographique à cette construction de Ferron du curé proche de ses paroissiens dans la synchèse précédemment citze de Fernand Dumont: «[Le prêtre] sait du latin, mais il est peu instruit en matière religieuse. De sorte que, dans sa predication, dans les conseils qu'il distribue au jour le jour, il renvoie à ses ouailles des opinions, des idées, des stéréotypes d'un milieu dont il est partie prenante au lieu de le dominer. Il est le relais d'informations qui lui viennent de plus loin; il est le porce-parole de sa communauté au moment même où il paraît luı dicter sa conduiten, Genese de le socuté québecosse, op. ctt., p. 234-235. A ce portrait qui convrent à la perfecrion au chanoine Tourigny, on opposera les développernents théologiques, "peut-être hététique[s] * (Le Saint-Élias, p. 100), de P'abbé Lupien.

38. Jacques FerRoN, Le Saint-Elhas, p. 28.

39. a[L'abbé Lupien] fasair partie des oiseaux rares pour qui la téalité ne compte guère, a laquelle ils ne se soumetront jamais et qui ne cesseront pas par contre, tout au cours de leur vie, de se la soumettre, soit par leurs idées, soit par leur pouvoir. Ils deviennent des arcistes, des saints, des fous, des criminels, quand us parviennent à vivren (Le Saint-Elias, p. 50). 
Cette opposition fait également écho à celle, chère à Dumont, entre les cultures première et seconde, l'une rattachée au monde de l'enfance et à la langue maternelle, à la cohérence du monde, et l'autre, à la "stylisation" qui permet au sujet de "prendre distance vis-à-vis d'elle et d'avoir conscience de sa signification d'ensemble ${ }^{40}$ ». Car entre le chanoine Tourigny et l'abbé Lupien, ce n'est pas d'une opposition entre deux cultures dont il faut parler; la première rattachée à la mémoire du pays et la seconde, universelle et complètement détachée du contexte où elle sévit. Il s'agit plutôt de l'isolement progressif d'une parole qui, prenant de la distance, peu à peu se perd. Lorsque Ferron écrit à Julien Bigras, revenant sur sa théorie du "moi crucifiant", avoir "conçu la religion comme un système syntaxique ${ }^{41}$ ", il témoigne peut-être surtout de la nécessité du syntagme, disons de la totalité de la phrase lui permettant de réunir le "seul" pronom personnel de ses acolytes "représentatifs ${ }^{42}$ \%. Il rend compte à la fois du besoin et de la difficulté pour l'écrivain de franchir sa solitude constitutive afin de donner sens à la communauté qu'il contemple à distance ${ }^{43}$. Ce qui étonne, c'est que ce "mécréant" ait conçu la syntaxe de l'intérieur du catholicisme, cette "religion nationale ${ }^{44}$ ", et qu'il ait eu besoin de passer par ce régime de signes particulier afin de pouvoir communiquer avec le monde ${ }^{45}$. Voilà précisément l'avantage de la tradition, au sens que lui donne Gershom Scholem en parlant de la mystique juive ${ }^{46}$, soit de pouvoir prêter ses signifiants usuels à la médiation d'une expérience inédite susceptible d'en renouveler la signification. Et, sans vouloir gloser indûment sur une "théorie» aussi personnelle que "métaphorique»,

$$
+4
$$

40. Fernand DUMONT, Le lieu de l'homme. La culture comme distance et mêmoire, Montréal, Bıbliochèque québécoise, [1968] 1994, p. 75 .

41. Julien Bigras et Jacques Ferron, Le desarroi. Correspondance, Montréal, VLB, 1988, p. 105.

42. Parmi rous les pronoms, un seul est personnel: le JE er ses adjoints. Les autres sont représentatifsn ( $D u$ fond de mon arriere-cutsine, p. 144).

43. N'oublions pas, à cet égard, l'une des dernières phrases du roman, visant à définir certe solitude de l'écrivain... et peur-être le pouvoir qui lui est imparti: "On écrit seul comme un roi» (Le Saint-Élias, p. 150). Voir également la conclusion d'un texte important de Ginette Michaud, qui commente ainsi le rapport à la langue de la mère de Ferron, gauchie par le fantasme du père qui le pousse à "écrite à l'anglaise" : "Ne plus écrire pour sa mère - il s'agıt bien indifféremment de la mère naturelle ou de la langue mère -, n'est-ce pas la plus haute trahison, celle pourtant que doit commettre tout écrivain, s'il veut, paradoxalement, accomplir son désir? ", "Lire à l'anglaise", Lautre Ferron, op. cit., p. 196.

44. uJe ne suis pas croyant, mais j'à toujours été curieux de savoir si c'était le Québec qui faisait la force de la religion ou si c'était la religion qu faisaic la force du Québec. Je me suis rendu compte à la fin que c'écaic notre pays qui donnait force à cette religion qui a été quelque temps une religion nationalen, Par la porte d'en arrizre, op. cit., p. 172.

45. Voir Le désarroi, op. cit., p. 104-105. Voir également ce qu'il affirme dans le "jeu des questions er des réponses» qui ouvre le livre de Jean Marcel: $*$ Je ne suis même pas athée. Je me prétends mécréant, c'est-d-dire en marge d'une religion et dépendant d'ellen, Jacques Ferron malgré lui, Montréal, Parti pris, 1978, p. 23. C'est bien sûr le dernier segment de la proposition qui importe, et sans doute fallait-il quelqu'un en margen du catholicisme pour ne pas céder à la tencation de la table rase qui emporta ses contemporains, et pouvoir ainsi se réclamer d'une certaine dépendance à son égard.

46. Voir Gershom SCHOLEM, La kabbale et sa symbolique, Paris, Perice Bibliothèque Payor, 1989, p. 31. 
comme nous l'avons vu, force est de constater que c'est encore elle, avec sa proposition politique structurant l'antique dialectique de l'un et du multiple, qui informe la figure centrale de la filiation du Saint-Élias.

"C'est le Fils qui a engendré le Père, et le Père par reconnaissance a créé pour nous la voûte des cieux ${ }^{47}$." Cet énoncé sur la filiation, aux atours théologiques - même s'il se rapproche davantage de la Gnose que d'une théologie proprement catholique - constitue le véritable centre du roman. Non seulement le retrouve-t-on à trois reprises ${ }^{48}$, mais c'est encore à partir de cet énoncé qu'il nous est possible de comprendre l'enjeu central de la filiation symbolique, plus particulièrement la question du nom sur laquelle se termine l'œuvre. D'ailleurs est-il nécessaire d'insister? Cette réflexion sur le nom est à ce point évidente qu'elle nourrit une partie de l'intrigue dans l'opposition de l'abbé Lupien et du marchand Philippe Cossette entre paternité biologique et paternité symbolique, ce qui n'est pas sans rappeler les développements joyciens sur la "fiction légale de la paternité "99". Mais les choses ne sont jamais aussi simples qu'elles le paraissent, chez Ferron, et si le petit Armour Cossette est le fils biologique d'Armour Lupien, il en porte aussi le prénom, tout en prolongeant la lignée des Mithridate, que l'abbé Lupien a lui-même consacrée en attribuant ce sobriquet au père symbolique, ou patronymique, Philippe Cossette.

Lélément essentiel de l'énoncé théologique de l'abbé Lupien est évidemment l'inversion qu'il met en branle. Plus qu'un simple refus de la tradition ${ }^{50}$ traduisant "l'indéfinie liberté» d'un sujet en mal de commencement, il me semble qu'il faut avant tout $\mathrm{y}$ voir un renversement du rapport à l'origine, voire l'invention d'une origine qui fait défaut. Ce renversement nous met aux prises aussi bien avec un désarrimage temporel - la référence au temps hors de ses gonds (out of joint) d'Hamlet et à la posture du Fils chargé de le remettre est ici transparente - qu'avec une figure de la langue.

$$
++4
$$

47. Jacques Ferron, Le Samt-Elias, p. 54.

48. Voir ibid, p. 9, 149.

49. James JOYCE, Ulysse. CEuvres II, Parıs, Gallimard, coll. " Bibliothèque de la Pléııden, 1995, p. 235.

50. Éric Méchoulan, interprétant l'énoncé sur la filiation comme étant une forme de "monarchie électiven (c'est le fils qu fait le père, de même que c'est l'écrivain qui choisit ses pères en littérature), conclut annsi sa lecture du Sasnt-Elias en ecrivant que la usolicude de l'ecriture" est une a solitude nombreuse [...], peuplée d'ombres choisies*, voir " "On écrit seul comme un roi". Le Saint-Elias de Ferron et le Saint Genest de Rotroum, Jacques Ferron; le palimpseste infini, op. cit., p. 203. 
Car cette question de l'origine à inventer fait aussi retour sur le statur de la parole et de son inscription, nécessairement historicisée, dans le champ toujours préexistant des noms. Aussi faut-il d'abord, avant d'entreprendre l'analyse des implications (épistémologiques et politiques) d'un tel énoncé, s'attaquer aux multiples figures chargées de lui donner sens. La première figure emprunte elle-même divers visages, puisqu'on la retrouve aussi bien sous les traits d'une "grande idole peule" aux "seins globuleux" et à la "face plate à la bouche béante, armée de dents terrifiantes ${ }^{51}$ " qu'en tant que "jeune femme, morte et toujours vivante", figure littéralement fantomatique, puisque celui qui cherche à se la remémorer demeure toujours dans l'«incapacité de donner une forme à son souvenir ${ }^{52}{ }_{n}$. La figure de la mère multiforme est d'autant plus fuyante qu'elle épouse les contours de nombreux personnages; de la mère de l'abbé Lupien, morte lorsqu'il avait trois ans, à Marguerite, son amante d'un jour et mère du petit Armour Cossette. Elle est cependant toujours le siège d'une origine incertaine, qu'elle soit liée au métissage (Marguerite) ou perdue dans l'oubli le plus reculé. En raison de cet oubli et de l'indétermination indélébile qui la marque, cette figure de la mère est appelée, chez Ferron, à servir de lieu imaginaire où reconstruire cette origine qui fait défaut. Le meilleur exemple d'une telle construction agit d'ailleurs comme une mise en abyme au sein du roman. Il s'agit du petit roman "dit d'action et d'aventures" que le jeune abbé Lupien, alors séminariste, avait écrit pendant ses vacances et qui raconte entre autres choses le sauvetage inusité de la fiancée du héros, emportée par le "courant» d'un lac et prisonnière de ses "remous». Mais cette Estelle n'est pas aussitôt "sauvée des eaux [qu'elle] allait bientôt mourir comme sa mère était morte, de la poitrine ${ }^{53}$ ». Ce premier sauvetage, commente alors le narrateur, «marquait justement son incapacité de le faire [...] Il n'était parvenu à l'évoquer que pour la reperdre, que pour mieux se signifier qu'elle resterait toujours perdue ${ }^{54} \%$. Que peut bien signifier cette signification elle-même? Quelle fonction occupe ce récit d'une rédemption manquée au sein du Saint-Elias? Cette mère, impossible à restituer, se peut-il qu'elle serve surtout à camoufler l'impossible restitution d'une entrée en langue du sujet, son inscription dans la chaîne des noms par l'acte même de la nomination, comme on le sait lourd de sens pour l'écrivain lui-même? Elle revêt en tous les cas à la perfection les habits de l'un des tout

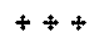

\footnotetext{
51. Jacques Ferron, Le Samt-Elias, p. 110.

52. lbid, p. 73 ex 75 .

53. Ibid, p. 77.

54. Ibid
} 
premiers spectres de la littérature occidentale, cette mère rencontrée par Ulysse au cœur de l'Hadès qui ne peut que repousser ses tentatives répétées de l'atteindre (et l'étreindre). Dans l'œuvre de Ferron, la mère serait ainsi une figure fantomatique qui ne saurait plus se faire entendre que par son silence d'outre-tombe; silence de l'Autre d'autant plus probant que c'est par son entremise que la parole du sujet procède. Ginette Michaud, citant Derrida, propose une interprétation qui va dans ce sens lorsqu'elle évoque l'écriture «à l'anglaise" de l'écrivain cherchant à se frayer un "passage hors la langue maternelle" : "Tel nous apparaît aujourd'hui Ferron: non pas "père" de la littérature québécoise moderne, mais éternel fils, d'une "génération nécessairement seconde, originairement tardive et dès lors destinée à hériter", qui a dû, comme Hamlet, "s'expliquer avec du spectre", avec plus d'un spectre ${ }^{55}$." Cette figure maternelle évanescente est aussi ce qui, d'une certaine manière, vient trouer la trame existentielle du sujet, cette "succession des jours" que "Dieu le Père" est appelé à tresser, surface blanche, littéralement, sur laquelle se succèdent les multiples apparitions chargées d'en "masquer" le vide aussi bien sur la surface du visible que dans l'ordre symbolique ${ }^{56}$.

Parmi ces multiples "masques» du roman, celui de Marguerite me semble particulièrement important afin de comprendre le rôle stratégique de l'énoncé sur la filiation. En vertu de la fièvre qui s'apprête à l'emporter, l'abbé Lupien passe ses derniers moments à dialoguer avec quelques-uns de ces succédanés. Parmi eux, nous retrouvons la figure de Marguerite, qui lui avoue qu'un autre Armour, son fils, lui a rapidement succédé et qui lui demande s'il aurait préféré qu'elle le lui sacrifie. Voici ce que l'abbé Lupien lui répond alors, soulignant par ces ultimes paroles le rôle de l'évanescence maternelle dans la discontinuité de son propre rapport au temps: «Non, Marguerite, il

$$
\div 4
$$

\footnotetext{
55. Ginetre MiCHAUD, „Lire à l'anglaisen, op. $c t$, p. 196-197. Le passage de Derrida qui est cité provient de Spectres de Marx, Paris, Galilée, 1993, p. 46. On a vu précédemment comment cetre posture de Fils dépasse le strict cadre romanesque du Saint-Elias. Il est d'autant plus troublant de vorr l'insıstance de certains écrivains, ou critiques, à faire de Ferron ce père de la moderniré romanesque québécoise. Je pense surtout ici à Vicror-Lévy Beaulıeu, qui n’a cessé de réclamer pour son propre compre cetce posture de fils qu'l subtilise à Ferron, avant de le sommer de reconnaître sa paternité symbolique. Voir, entre autres cextes, la dernière de ses lettres à Ferron, récemment publı́es: «Mol, je sals bien que je n'ai que du calent, mais un calent forcené, qui ne lâchera jamais, ne serait-ce que pour saluer le génie de mes pères, celui de mon géniteur er le vôtre [...] vous êres ce qui nous est arrivé de mieux et, puisque vous ne semblez pas le comprendre, I faudra bien que nous occupions votre place, pour crier après vous", Victor-Levy BEAULIEU et Jacques FERRON, "Lettre du 15 septembre 1983", Correspondances, Trois-Pistoles, Editions Trors-Pistoles, 2005, p. 92-93.

56. "Sur le lir donr il ne se relèverait pas, dans sa chambre du pauvre p'it presbytère de Saint-Thuribe, des femmes se penchaient sur l'abbé Armour Lupien. C'était Isola, sa ménagère, c'était une petite bonne dont il ne connaissair pas le nom, c'érait Estelle, c'étatt Marguerite. Detrière tous ces visages, il en cherchait un autre dont il ne se souvenatc pas, celui de sa mère qu'il avart perdue alors qu'il venait d'avoir troıs ans, qu'il avaic oubliée mais dont le visage devait se trouver derrière tous ceux qu'il voyait et qui n'en étaient peut-être que les masques" (Le Samt-Eltas, p. 73). Voir aussi p. 79, où Isola, entendant l'abbé evoquer ces noms de femmes, trouve «naturel" que ces divers masques servent $d^{\prime}$ ' apput a la "Vierge Mère quand elle se penche sur son Fils*.
} 
faut l'aimer; il faut recoudre en lui la succession des jours qui chez moi s'est rompue. Je n'étais qu'un chien errant qui trânait un bout de corde qu'une femme obscure avait commencé de tresser ${ }^{57}$." Au-delà de l'anecdote directement biographique concernant aussi bien l'abbé Lupien que l'écrivain Ferron lui-même (et sa "mère cadette»), il faut surtout retenir de ce passage la proposition sur le rôle (linguistique et temporel) de la mélancolie. On se souviendra que Freud fait de la mélancolie le retournement sur le moi de la libido retirée de l'objet aimé, créant ainsi une "identification" du moi et de cet objet qui à son tour entraîne une forme de régression ${ }^{58}$. Nous pouvons résumer la proposition de Ferron, complémentaire à celle de Freud, de la façon suivante: conséquemment à la disparition de l'objet d'amour par excellence - mais qu'il aurait tout aussi bien pu anticiper -, le mélancolique défend la nécessité de tisser de son propre chef la corde, ou la "tresse", qui le relie au monde. En termes linguistiques, cela signifie trouver la langue maternelle en défaut ${ }^{59}$, invoquer le Verbe (ou le Père) afin de pouvoir s'inscrire dans la chaine des noms de son propre nom (ou chef), c'est-à-dire en Fils unique "mourant sur le Calvaire ${ }^{60}$ " et "réengendrant" pour soi la langue (le Verbe) permettant la lecture (l'appréhension) du monde. C'est créer une légère "fêlure" dans la langue afin de s'y inscrire, non par extravagance mais en la dédoublant, en mettant de l'avant sa nature linguistique pour ellemême, sans finalité (de communication) ni visée instrumentale. C'est, en termes ferroniens, réinjecter du Père (du Verbe) dans le Fils, c'est-à-dire mettre de l'avant la nature essentiellement linguistique du sujet et la nécessaire interprétation de la langue qui accompagne un tel constat. "Pervertir la forme [du langage] pour en couler le fond ${ }^{61}$ " ne veut pas dire autre chose. Loin de l'expérimentation exploréenne, que Ferron ne connaissait que trop bien ${ }^{62}$, il s'agit de souligner la forme au détriment du contenu linguistique, de montrer, littéralement, la parole en acte, et d'en faire un théâtre, suivant l'exemple de

$$
+4
$$

57. Jacques Ferron, Le Saint-Elias, p. 82.

58. Voir Sigmund FREUD, «Deuil et mélancolien, Metapsychologie, Paris, Gallimard, coll. "Folion, 1991, p. 145-171. Relisant le texte de Freud à partir d'une analogie avec la figure, familière aux Pères de l'Église, de l'acedia, Gıorgio Agamben soutient que la mélancolie " traduit moins une éclipse du désir que la mise hors d'atteinte de son objet \#. Cette proposition est intéressante pour son ouvercure du désır à la dimension temporelle, à un à venir toujours présent parce que toujours reporte. Voir Giorgio AGAMBEN, Stanze. Parole et fantasme dans la culrure occidentale, Paris, Rivages, «Petite bibliothèquen, 1998, p. $25-26$.

59. Dans l'un des conres de La conference inachevé, „Le Chichemayais», Ferron écrit: «Ma mère subsistait comme une divinité dont la langue éait trop belle pour avoir cours. s (La conference inachevée, p. 105).

60. Jacques FerRon, Le Saint-Eltras, p. 99.

61. Jacques FerRon, La conftrence inachevee, p. 209.

62. Voir, avec "Les salicaires", le texte "Claude Gauvreau", dans Du fond de mon arrikre-cuisine, p. 201-264, où Ferron relate sa rencontre avec Gauvreau, de même que les nombreux š́jours de celui-ci en psychiatrie. 
l'extraordinaire cérémonie d'adieu - les funérailles religieuses étant exclues par le suicide - préparée par l'abbé Tourigny pour son ami le docteur Fauteux. Un théâtre de gestes et de chants afin de montrer l'ultime parole du médecin de campagne, qui a pris sur lui la faute qui a conduit l'abbé Lupien à la mort et l'infamie des "fous et [d]es pauvres misérables dans le champ du Potier ${ }^{63}$ ". Mettre de l'avant la "syntaxe" et le Verbe, voilà somme toute le sens de l'énoncé de prime abord cryptique de l'abbé Lupien: "C'est le Fils qui a engendré le Père et, sans Jésus mourant sur le Calvaire, il n'y aurait pas de Dieu ${ }^{64}$. " Un sens de nature politique puisqu'il ne saurait $y$ avoir d'expérience du commun sans prendre en compte la manière de cette expérience, sans un usage de la langue qui dans sa singularité définit par lui-même le rapport qu'entretient le sujet avec l'espace public (et politique).

\section{LANGUE OUVERTE ET TEMPS SUSPENDU: LE SOLEIL ET LES GABAONITES}

Dans une parenthèse des plus étonnante - d'autant plus qu'elle revient à deux reprises dans les dernières pages du Saint-Élias -, Ferron soutient erronément que Samuel Butler aurait écrit Erewhon, anagramme de nowhere, alors qu'il se trouvait à Montréal ${ }^{65}$. Outre la corrélation entre le résumé laconique qu'en donne le roman - «Samuel Butler [...] a écrit un livre intitulé Erewhon où un peuple heureux garde en musée toutes les machines qu'il a inventées et dont il a été assez sage pour ne pas se servir ${ }^{66}{ }^{\prime}$ - et la critique du monde de la technique qui clôt d'une manière imprévisible Le Saint-Élias, il existe une signification encore plus déterminante de cette parenthèse. En insistant sur le lieu d'écriture d'Erewhon, Ferron insinue que Montréal, et par conséquent le Québec tout entier, aurait pu servir à Butler de modèle. Il fait ainsi implicitement du Québec le lieu d'une utopie, soit une entité politique imaginaire, n'ayant du moins jamais eu cours dans l'histoire. On ne ferait sans doute nullement attention à cette référence si un autre discours utopique n'était pas sous-jacent à l'ensemble de l'œuvre. Je pense ici à la querelle entre le libéralisme et l'ultramontanisme, représentée dans le roman par l'entremise des personnages du docteur Fauteux, anciennement du parti des Patriotes et maintenant de l'Institut canadien ${ }^{67}$, et de Mgr Laflèche, évêque de Trois-

$$
++
$$

63. Jacques Ferron, Le Samt-Elias, p. 109.

64. bid, p. 99.

65. La note 81 de l'édition Typo nous apprend que le roman fut publié en 1872, alors que Butler a séjourné au Canada en 1874-1875.

66. Jacques FerRon, Le Samt-Elias, p. 131.

67. lbid, p. 39. 
Rivières et fervent ultramontain, qui selon Fernand Dumont fut l'un des premiers à donner une "teneur strictement religieuse» à la "mission providenrielle» de la nation canadienne: "l'établissement d'un peuple profondément catholique dans cette terre que [la Providence] leur a donnée en héritage ${ }^{68}$ ". Mgr Laflèche allant jusqu'à comparer Jacques Cartier à Abraham, "tous les deux prenant possession d'une terre nouvelle ${ }^{69}{ }^{\prime}$. S'agit-il d'une simple coïncidence ou existe-t-il un lien entre cette allusion au Québec comme modèle d'un lieu de Nulle part (nowhere) et la présence du "messianisme canadien-français ${ }^{70}$ " en arrière-plan de l'œuvre? Même si le personnage de Mgr Laflèche est dépeint sous un jour défavorable, se peut-il que le roman, par sa seule présence, fasse signe vers un élément de ce discours messianique qui serait à conserver? En d'autres mots, la question qu'il faut se poser, c'est si la référence à l'utopie au sein du Saint-Élias n'indique pas la permanence, ou la résurgence, d'une structure de l'utopie, notamment temporelle, à défaut de son contenu manifeste - celle de la vocation d'une présence catholique en terre d'Amérique.

Au-delà des notions de "destin" ou de "Providence», voire de tout élément directement religieux, ce messianisme caractéristique de la "survivance" impliquait surtout la promesse d'une Rédemption future, devant nécessairement surgir comme réparation d'un présent jugé catastrophique. $\mathrm{Si}$ l'on enlève du discours messianique la pointe religieuse de la Providence, il reste avant tout une structure temporelle qui définit le présent sur le mode de l'attente. Une attente d'autant plus prégnante que le présent qui sévit se retrouve indéfiniment maintenu, privé de l'horizon qui était chargé de lui donner sens.

Vers la fin de sa vie, Ferron a évoqué à quelques reprises une figure biblique qui, selon lui, représentait bien la situation du Québec. Il s'agit du peuple des Gabaonites, dans le Livre de Josué, qui serait à l'origine des qualificatifs de "porteurs d'eau" et de "scieurs de bois" souvent appelés à définir les Canadiens français ${ }^{71}$. Pour mémoire, ces Gabaonites sont des

$$
+4
$$

68. Louis-François LAFLECHE, Quelques considerations sur les rapports de la socteté civile avec la religion et La famille, Montréal, Eusètbe Sénécal, 1866, p. 37-38, cité par Fernand DUMONT, Genèse de la société québecoise, op. cit.. p. 273. 69. Ibid

70. Sur le «messianisme canadien-français”, outre le chapitre sur ale recours a l'utopien du livre de Fernand Dumont, voir Michel BRUNET, "Trois dominantes de la pensé canadıenne-française: l'agrıculturisme, l'anti-ktatisme et le messianisme", La presence anglaise et les Canadiens. Etudes sur l'bistoire et la pensée des deux Canadas, Montreal, Beauchemin, 1964, p. 113-166; et Rejean BEAUDOIN, "Considérations sur le messıanisme canadien-français», Écrio du Canada français, $n^{\circ} 53,1984$, p. $105-125$.

71. Voir Par la porte d'en arrière, op. cit. p. 130: «Je n'ai pas beaucoup fréquenté la Bıble, mais hier j'ai lu ce livre d'horreurs que peut être l'Ancien Testament, en particulier le "Livre de Josuế" [...] Je me suis rendu compte d'une chose: 
habitants de la terre de Canaan, et de ce fait "voués à l'interdit ${ }^{72}$ ", c'est-à-dire à la mort, par la Loi de Moïse. Ils emploient donc la ruse et se présentent devant Josué comme des nomades, habillés de haillons et munis de pain sec, venus d'une contrée lointaine afin de leur prêter allégeance. Les responsables d'Israël font alors le serment de les protéger et recourent à leurs services comme "fendeurs de bois et porteurs d'eau». Le peuple d'Israël prendra même les armes pour les défendre après avoir découvert la vérité, bataille au cours de laquelle Josué arrêta le soleil "pendant près d'un jour entier ${ }^{73}$ " pour se venger de ses ennemis. La conclusion de Ferron, éminemment politique, est des plus intéressante puisque sa lecture du récit biblique opère un renversement par rapport à la tradition messianique qui fait des Canadiens français un peuple porteur d'une vocation: c'est au peuple de Gabaon qu'il identifie ainsi celui du Québec, peuple de porteurs d'eau et de scieurs de bois, comparant du même coup les Anglais aux Israélites, en soulignant au passage l'aspect "liturgique" de la langue anglaise:

Ça nous donne une situation de dépendance sanctionnée par les livres saints [...] L'Amérique peut être considérée comme une Terre promise par les nouveaux arrivants qui sont évidemment le peuple élu de Dieu, et nous, on nous permet de survivre, on ne nous passe pas au fil de l'épée, on ne tue pas nos enfants ${ }^{74}$.

Ce qui me semble intéressant dans ce commentaire de Ferron, c'est le renvoi au texte biblique afin de revivifier le sens d'une situation historique de dépendance. Voilà le parfait exemple d'un recours à la tradition en tant que tradition, car s'il importe peu à Ferron de rester fidèle à l'analogie avec le peuple juif, telle que déployée dans le messianisme canadien-français de la "survivance", la référence au Livre de Josué et à sa célèbre figure, parfaitement messianique, nous montre bien comment la transmissibilité propre à la tradition continue à opérer dans son œuvre. Sa description de l'Amérique comme nouvelle "Terre promise", mais où les Québécois n'auraient qu'un rôle passif, va tout à fait dans le sens d'un rapport utopique à l'histoire où l'utopie elle-même qui en est à l'origine serait privée de signification; une

$$
+4
$$

nous écions dans la sicuacion des Gabaonites. Ce sont des porreurs d'eau er des scieurs de bois. Je savais qu'on nous avait comparés à des porteurs d'eau et des scieurs de bois, mais je ne savais pas avant hier que ça fassait allusion à une page biblique."

72. Voir le «Deutéronome», 20, 16-18.

73. "Livre de Josué», 10, 13.

74. Par la porte d'en arriere, op. ctt., p. 131. 
histoire dans laquelle le soleil aurait suspendu sa course, en attente d'un événement susceptible de la relancer.

En ce sens, le rapport à la tradition chez Ferron est comparable à celui qui a cours dans l'œuvre de Kafka selon l'interprétation de Gershom Scholem et de Walter Benjamin, comme l'a d'ailleurs déjà noté Alexis Nouss $^{75}$. Mais il n'y a bien sûr rien d'étonnant à cela, dans la mesure où l'on a souvent affirmé que l'œuvre de Kafka refléterait la crise de la tradition qui surgit dans l'Europe de l'entre-deux-guerres, alors que Ferron se ferait l'écho de celle issue de la Révolution tranquille. Si Benjamin affirme que l'œuvre de Kafka "présente une tradition tombée malade", il souligne en contrepartie la persistance de ce rapport à la tradition, le fait qu'il «renonça à la vérité pour ne pas lâcher la transmissibilitét ${ }^{76}$. De la même façon, nous pouvons dire que Ferron renonce au contenu de l'utopie messianique, mais sans renoncer à sa structure, qui elle demeure intacte. Cette question de la tradition va d'ailleurs bien au-delà de sa transmissibilité; elle trouve son fondement dans un rapport à la langue qui nous permettra d'identifier la conception de l'histoire dans laquelle s'inscrit cette structure temporelle de l'utopie.

À Benjamin qui lui demande son interprétation du Procès, Scholem écrit: "nulle part encore, la lumière de la Révélation n’a brûlé de façon aussi impitoyable qu'ici. Voilà le secret de la prose parfaite ${ }^{77}$." Il faut savoir que pour celui-ci, suivant en cela la conception traditionnelle de la cabale, la Révélation n'est pas transmission de la Loi, mais dans un premier temps dépourvue de toute signification Révélation de la Voix transcendante, c'est-à-dire d'une voix pourvue de sens, mais dont les significations resteraient indéfiniment à pourvoir. C'est le sens qu'il faut attribuer à la conception de la Loi, chez Kafka, qui tout à la fois demeure en vigueur, mais ne signifie pas, Scholem voyant à l'œuvre dans Le procès un "néant de la Révélation ${ }^{78}$ " (Nichts der Offenbarung). Pour l'historien de la cabale, le caractère inaccessible de la Loi ne signifie cependant pas que celui qui est

$$
+4
$$

75. Alexis Nouss, "Ferron et Kafka : le texte flottant", Litttratures, $n^{\infty}$ 9-10, 1992, "Présence de Jacques Ferron ", p. 113126.

76. Lettre du 12 juin 1938 de Walter Benjamin a Gershom Scholem, Walter BENJAMIN, Correspondance, tome Il, 1929 1940, édition établie et annorée par Gershom Scholem et Théodor W. Adorno, Paris, Aubier Montaigne, 1979, p. 251. 77. Letre du $1^{\text {to }}$ aût 1931 de Gershom Scholem à Walter Benjamin, Gershom SCHOLE, Walter Benjamin. Histoire d'une amitze, Paris, Hachette, coll. « Plurieln, 2001, p. 194.

78. "Tu me demandes ce que j'entends par "néant de la Révélation". J'entends par là un érat dans lequel celle-ci se manifeste comme vide de toute significacion, c'est-à-dire où elle continue à s'affirmer, où elle conserve sa validité, mais où elle ne signifie pas», Walter BENJAMIN et Gershom SCHOLEM, Briffuechsel 1933-1940, Francfort-sur-le-Main, Suhrkamp, 1980, p. 175, cité par Stéphane MOSts, L'ange de l'hustoire. Rosenzuweig, Benjamin, Scholem, Paris, Seuil, coll. - La couleur des idtes", 1992, p. 223. 
appelé à en témoigner puisse dire n'importe quoi. Le commentaire, pour être valide, se doit au contraire de répondre à un impératif: celui de se situer par rapport à la tradition. Il en va de même pour la langue. On comprend que Lacan, alors en pleine "relecture de Freud", ait pu à un certain moment s'intéresser à Heidegger, dont il a même traduit l'un des Essais et conférences. C'est que dans sa définition du Dasein comme "être-au-monde", Heidegger articule une conception du sujet comme "être-jeté" qui correspond bien à la situation du sujet de la psychanalyse qui est d'abord et avant tout un sujet du symbolique, c'est-à-dire sujet d'une langue qui lui préexiste et dans laquelle il a à s'inscrire; une langue à qui il est contraint de dire oui.

En cautionnant, en acceptant de raconter - ou en créant de toutes pièces, ce qui ici revient au même -, l'ultime injonction maternelle qui est de changer son prénom de Jean-Jacques à Jacques, Ferron fait écho à cette conception du sujet comme être-jeté dans la langue. Il accepte de se soumettre à une sphère des noms qui lui préexiste, ce qui définit par excellence l'historicité de la langue - cette langue maternelle toujours déjà pourvue d'histoire avant même l'avènement du sujet. L'auteur s'inscrit ainsi dans une tradition qui, loin de limiter la liberté qui lui est propre, lui ouvre toutes grandes les portes de ce que l'on peut nommer limaginaire de la généalogie. Ainsi, lorsqu'il appuie le récit du Saint-Élias sur les Annales du couvent des Ursulines de Trois-Rivières, qui dans ce cas précis se confond avec la chronique proprement familiale des Caron, sa famille maternelle, il se sert de ce matériau comme d'un support pour son interrogation de la filiation et de l'émergence de la folie, bref comme d'une trame sur laquelle ajouter une autre série de fils, ceux-là proprement romanesques. Ce qu'il faut donc retenir, selon cette perspective, c'est comment Ferron a pu se servir d'un vocabulaire spécifique - ce lexique proprement théologique du Verbe incarné par le Fils, de sa mort solitaire sur la croix, etc. - pour mieux le pourvoir d'un sens qui à priori en est absent. Il en va naturellement ainsi dans son rapport au messianisme traditionnel, qui a pu servir de toile de fond au Saint-Élias, et de la structure temporelle qui lui est propre, repérable jusqu'au sein de certains des "contes d'adieu" de La conférence inachevée, notamment "Le Chichemayais" et "Les deux lys", dont j'aimerais, pour finir, souligner quelques traits pouvant contribuer à la définition éventuelle d'une historicité québécoise issue de cette relecture du messianisme.

Nous pouvons d'abord noter limportance de la remémoration au sein de ces contes qui, sur le modèle de la récapitulation finale, évoquent aussi bien la proximité de la mort que l'enfance la plus reculée. Nous retrouvons ainsi la figure du soleil des Gabaonites dans les prières d'un petit garçon: 
Josué du haut de son cheval arrêtant le soleil et Zénon d'Élée qui, mieux encore, sa flèche décochée, la laissait dans sa volée sans jamais rejoindre la cible, n'ont pas offensé à ma raison. Avant eux, j’avais déjà tenté d'arrêter le temps ou du moins de le fractionner à l'infini, pour ne pas me retrouver devant la porte du Jardin de l'Enfance $[\ldots]^{79}$.

Mais, au-delà de la présence explicite de ce temps suspendu, nous relevons également l'évocation de la mère du garçon décédée depuis peu, "divinité dont la langue était trop belle pour avoir cours ${ }^{80}$ ». Cette distance prise envers la langue maternelle entraîne alors un intéressant jeu sur les noms et les lettres qui les composent. Ce jeu prend la forme d'une conversation entre le jeune protagoniste et l'abbé Surprenant alors que le premier, victime du temps qui passe, est à bord du train qui le ramène au Jardin d'Enfance. Qu'il soit question de l'orthographe du patronyme Bellemare, du village Point-du-Lac ou du rang Vie-de-Poche, qui aura une histoire différente si on l'épelle VidePoche, en deux mots plutôt qu'en trois, l'ecclésiastique offre au petit Jacques une leçon d'herméneutique qui fait écho au rapport à la tradition que l'on a $\mathrm{pu}$ voir à l'œuvre au sein du Saint-Élias. Un rapport au nom, pour paraphraser Scholem, où celui-ci "conserve sa validité mais ne signifie pas", offrant tous ses possibles à celui qui choisit de s'en saisir pour le revivifier.

Cette figure fantomatique de la mère revient également dans le tout dernier conte du recueil, cette ultime récapitulation de l'écrivain qui évoque aussi bien sa "fin prochaine" qu'une enfance encore plus reculée, alors que sa mère vivait toujours. Sa présence ne sert cependant plus cette fois à interroger la langue, mais bien le temps lui-même, ce "temps du temps qui passe $^{81}$ ». En effet, au-delà de l'argument des deux lys, celui "barbouillé de rouge" qui fleurit pour la fête des Orangistes, le 12 juillet, et le "vrai", selon la mère, le blanc dont le "parfum s'exalte $\left[\right.$ sic] des temps anciens ${ }^{82}$ ", ce qui importe dans ce conte, c'est le contexte de la remémoration de la figure de la mère dont le "visage", écrit Ferron, se fait "plus indistinct d'une année à l'autre ${ }^{83}$ \%. Et ce contexte est d'abord temporel. La question qui ouvre le conte, "Seigneur, qu'adviendra-t-il de cette journée ${ }^{84}$ ?", donne ainsi l'occasion au narrateur de réfléchir sur la nature indécise du temps; soir
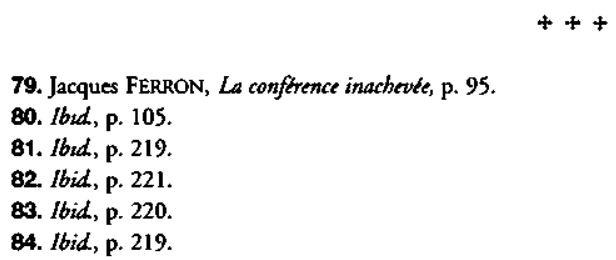
temporalité linéaire - ce "temps du temps qui passe" -, soit "temps qui n'est déjà plus le temps - ou qui ne l'est pas encore", qu'il identifie, par l'entremise d'une apostrophe, "au moment éternel et glorieux ${ }^{85}$ " de l'apothéose du Seigneur. Mais qu'est-ce donc qu'un temps situé entre un "déjà-plus» et un "pas-encore"? Il n'est pas indifférent qu'à cette interrogation sur le temps Ferron ajoute la question "Aurais-je vécu inutilement dans l'obsession d'un pays perdu ${ }^{86}$ ? ", si l'on tient compte du fait que la conception de l'histoire d'un sujet ou d'une nation repose avant tout sur la place occupée par ce sujet dans l'histoire. J'appelle temps messianique ce temps de l'intervalle entre le dejà-plus et le pas-encore pour insister sur la double détermination de cette historicité à l'œuvre dans La conférence inachevée: un temps qui est à la fois un temps de la remémoration, qui est tout autre chose que le souvenir, et celui de l'attente. Attente de l'inédit, si ce n'est de l'imprévu, qui malgré leurs divergences anime l'ensemble des messianismes qui ont eu cours aussi bien dans l'histoire que dans les traditions religieuses, et qui partagent une même conception de la rupture "entre le temps présent et l'ère messianique ${ }^{87}$ \#. Une appréhension de l'histoire qui se fonde sur le discontinu plutôt que sur le progrès, et qui marque peut-être le trait le plus sûr d'une historicité que l'œuvre de Ferron permet d'esquisser à même sa lecture de discours que l'on a pu croire révolus.

85. 1 ibid

86. $l b u d$, p. 222.

87. Gershom SCHOLEM, Sabbatai Tsevi. Le messie mystique, Paris, Verdier, «Les Dix Paroles ", 1983, p. 26 : " Aucune continuté n'existe entre le remps présent et l'ère messianıque; celle-ci n'est pas le frutt de progrès antérieurs ni d'une évolution graduelle. Loin d'ècre le résultar d'un processus historique, la rédemption s'élève sur les ruines de l'histoire qui s'effondre lors des "douleurs de l'enfantemenc" de l'àge messıanique. " 